\title{
Effect of microparticle properties on erosion in solid-steam mixtures
}

\author{
XU WanLi $i^{1,2^{*}}$, WANG JiaDao ${ }^{2}$, ZHAO Wei ${ }^{1,2}$ \& CHEN DaRong ${ }^{2}$ \\ ${ }^{1}$ Beijing Petroleum Oil and Lubricant Institute, Beijing 102300, China; \\ ${ }^{2}$ State Key Laboratory of Tribology, Tsinghua University, Beijing 100084, China
}

Received September 25, 2010; accepted January 5, 2010

\begin{abstract}
Based on a custom-built experimental apparatus, irregular microparticles of different sizes and microparticles of the same size but of different shape were added to wet steam, and erosion experiments were performed on polished medium carbon steel specimens. After the experiments, the eroded surfaces were analyzed by scanning electron microscopy and the degree of damage was evaluated by area loss. The results indicated that microparticle size significantly affects the degree of erosion damage. With an increase in size, variations in the method of damage mainly cause different degrees of damage on an eroded surface. Microparticle shape hardly affects the number of craters. Compared with spherical microparticles, irregular microparticles cause indentations more easily and result in more severe abrasion on the material's surface.
\end{abstract}

erosion, abrasion, microparticles, wet steam

Citation: $\quad$ Xu W L, Wang J D, Zhao W, et al. Effect of microparticle properties on erosion in solid-steam mixtures. Chinese Sci Bull, 2011, 56: 933-937, doi: $10.1007 / \mathrm{s} 11434-011-4385-3$

Erosion in steam turbine blades is an important problem in power generation systems [1,2]. It is commonly acknowledged that water drop impact is the main reason for damage on the surfaces of last stage blades and that the damage results from the direct impact of water drops [3-6]. Min et al. [3] and Mann et al. [4] performed water jet impingement experiments on solid surfaces and found that the initiation and expansion of the cracks occurs on the surface because of a stress wave and a high-speed lateral jet that is generated by water drop impact. However, microparticles exist in steam because ferric oxides peel from the inside of a superheated tube [7], which then changes the type of damage [8]. We have previously investigated erosion damage by wet steam that contained microparticles [9]. The indentations and scratches caused by microparticle impact and the cracks caused by water drop impact are not the only types of damage caused when the steel surface is eroded by wet steam that contains microparticles because craters also appear on the surface. Microparticles have been shown to have an

*Corresponding author (email: xuw106@mails.tsinghua.edu.cn) important role in the generation of craters and the craters are considered to be the result of cavitation erosion. Therefore, we studied the effect of microparticle properties on wet steam erosion.

As part of our ongoing study, erosion experiments using wet steam containing different microparticles were carried out with a custom-built experimental apparatus. According to the degree of material surface damage, the effects of microparticle size and shape on cavitation erosion and abrasion were investigated.

\section{Experimental section}

\subsection{Experimental apparatus}

Figure 1 shows a schematic diagram of our experimental apparatus. Wet steam is generated by the steam generator (A). Velocity changes and variations in the flow rate of the wet steam are controlled using valve (B). Microparticles are forced into the pipeline by the designed thruster $(\mathrm{H})$ after being dispersed by the supersonic vibration device $(\mathrm{G})$. The 
feed rate of the microparticles can be controlled by changing the rotary speed of the stepper motor. Wet steam carrying microparticles then impact the sample surface $(\mathrm{J})$ after passing through a mixer (C), steam flow meter (D), pressure transmitter (E), temperature sensor (F) and nozzle (I). After completion of the erosion experiment the pipeline can be cleaned with deionized water.

The sample is set in the jig (K). The distance between the sample and the nozzle and the included angle between steam impact and the sample can be adjusted by altering the position of the metal bars and rotating the jig's angle.

\subsection{Experimental procedures}

Two types of microparticle were added to the wet steam by the designed thruster. One type is irregular $\mathrm{SiC}$ microparticles and the other is spherical $\mathrm{SiC}$ microparticles. Figure 2 shows scanning electron microscope (SEM, FEI Quanta 200) micrographs of the microparticles. The spherical microparticles have smooth surfaces while crevices and concaves are found on the surfaces of the irregular microparticles. To determine the effect of microparticle properties the degree of erosion damage was evaluated. Many methods exist to evaluate the degree of erosion damage such as mass loss and pit number [10]. In our experiment, plastic deformation appeared on the surface and the shape and size of the erosion pits were different [9]. By contrast, area loss is

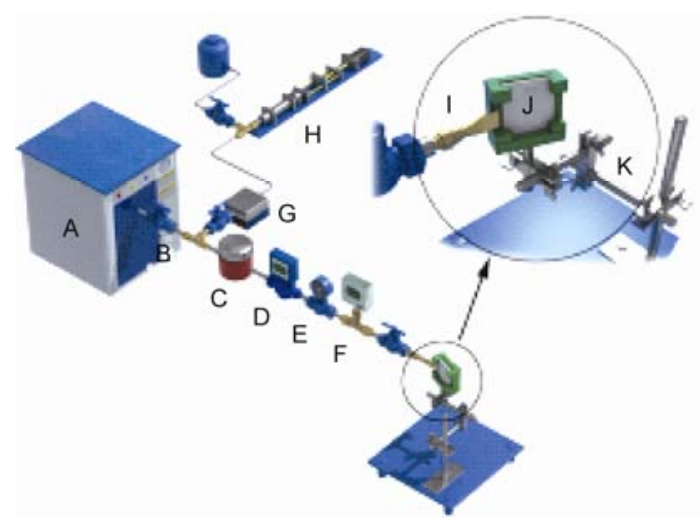

Figure 1 Schematic diagram of the experimental apparatus. more suitable to evaluate the degree of erosion damage [11]. Herein, the area loss is defined as the area of the indentations, scratches or craters that result from microparticle or cavitation erosion.

Specimens were made using medium carbon steel (AISI 1045 steel). The surface was polished and the mean square ( Rq) value was $50 \pm 5.6 \mathrm{~nm}$, which was measured by atomic force microscopy (AFM, CSPM 4000). Deionized water that contained a few microparticles was used as the steam source. To avoid material destruction by water-drop impact a steam velocity of $80 \mathrm{~m} / \mathrm{s}$ was used because the estimated damage threshold velocity for liquid impact is $120 \mathrm{~m} / \mathrm{s}$ [12]. Because the diameter of the nozzle is $2 \mu \mathrm{m}$, the impact velocity of steam can be controlled by steam flow. The other experimental conditions were as follows: steam pressure of $0.1 \mathrm{MPa}$ and a steam temperature of $110^{\circ} \mathrm{C}$, which was obtained using a pressure transmitter and a temperature sensor, respectively. The impact angle was $20^{\circ}$ and the impact distance was $10 \mathrm{~mm}$. During the $10 \mathrm{~min}$ experimental time, a total of $0.6 \mathrm{~g}$ of the microparticles were equally mixed into the steam pipeline through the designed thruster.

\section{Results and discussion}

\subsection{Effect of microparticle size}

Huang et al. [13] investigated the effect of solid particle properties on cavitation erosion in solid-water mixtures. Particle abrasion was found to be so severe that it was difficult to distinguish the damage caused by cavitation erosion. To differentiate the damage induced by abrasion and cavitation erosion irregular microparticles with nominal sizes of $0.5,0.8,1.0,2.5,3.5,5,6.5,8,9$ and $10 \mu \mathrm{m}$ were studied.

After the erosion experiments, the eroded surfaces were analyzed by SEM and Figure 3 shows the micrographs of some eroded surfaces.

Figure 3(a) shows that indentations and craters appear on the eroded surface. The indentations are triangular while the craters are circular. The indentation area is smaller than that of the craters. Figure 3(b) shows that the indentations and
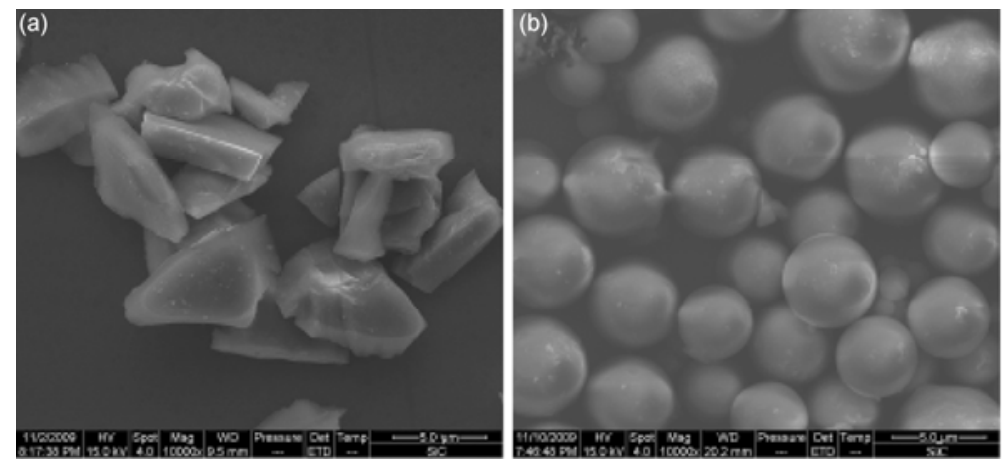

Figure 2 SEM micrographs of (a) irregular microparticles and (b) spherical microparticles. 

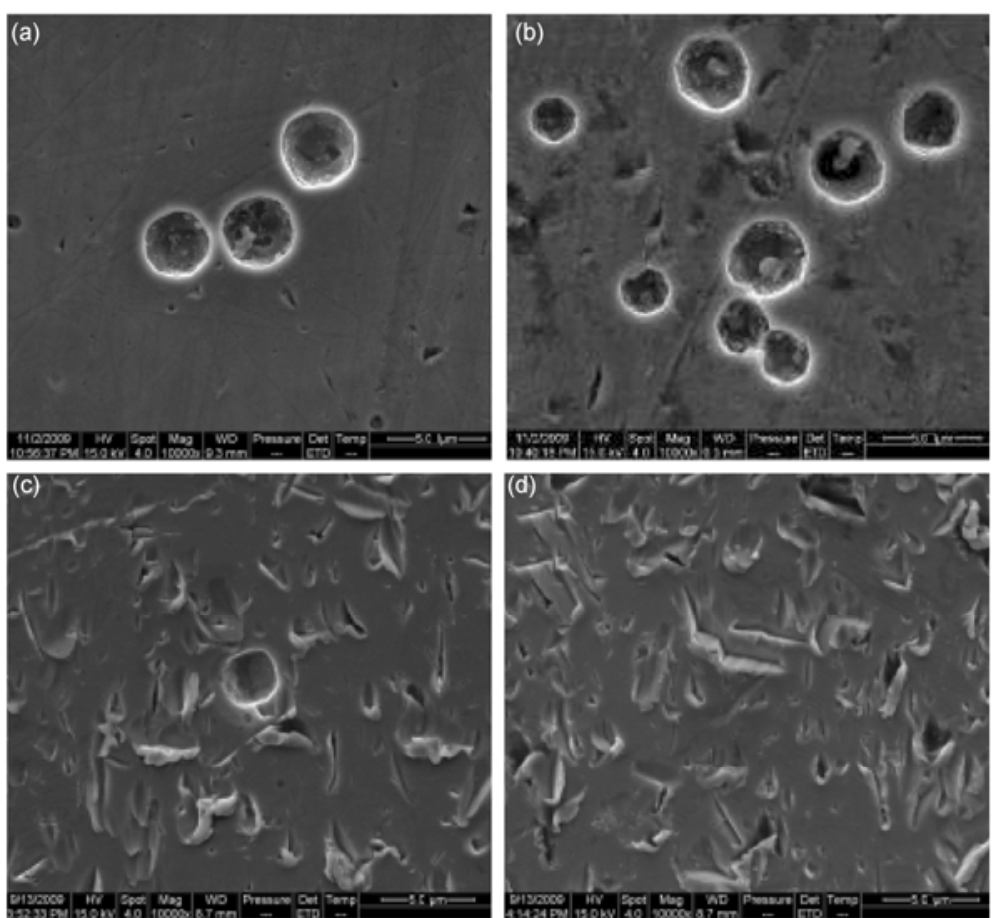

Figure 3 Scanning electron micrographs of surfaces eroded by adding irregular microparticles of different size to wet steam. (a) $0.8 \mu$ m; (b) $3.5 \mu$ m; (c) 6.5 $\mu \mathrm{m}$; (d) $8 \mu \mathrm{m}$.

craters also appear on the eroded surface, but the number of indentations and craters increase. Figure 3(c) shows that scratches appear on the eroded surfaces next to the indentations and the number of crater decrease. The scratches are strip shaped with lengths of $0.5-6 \mu \mathrm{m}$ and widths of $0.2-2$ $\mu \mathrm{m}$. When the $8 \mu \mathrm{m}$ microparticles were added to the wet steam the eroded surfaces had few craters and scratches are the main type of damage, as shown in Figure 3(d).

To obtain the areas of the craters, indentations and scratches, scanning electron micrographs were processed by pit identification including pit profile recognition, border connection and fill, and information statistics [11]. According to the statistical results a relationship between area loss and microparticle size was obtained, as shown in Figure 4.

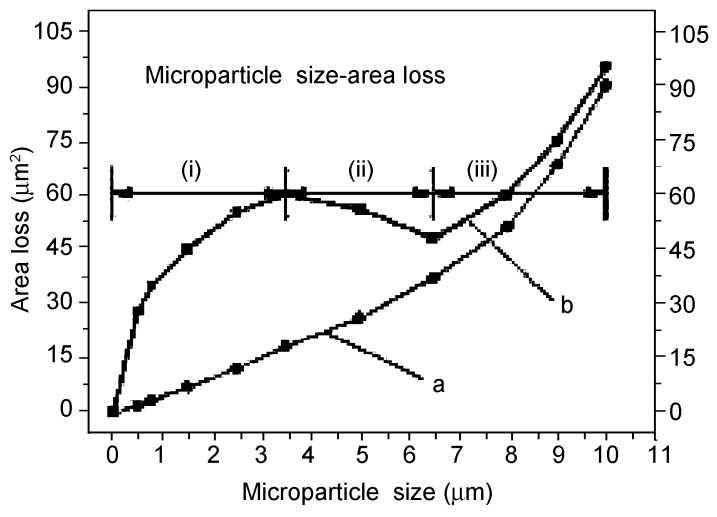

Figure 4 Relationship between area loss and microparticle size.
Curve a represents the area loss caused by indentations and scratches. The area loss increases with an increase in microparticle size, which also agrees with the report by Rickard et al. [14]. Curve b shows the area loss caused by indentations, scratches and craters. With an increase in microparticle size the total area loss can be divided into three parts: (i) A direct ratio I where the area loss increases rapidly. In this part, indentations and craters appear on the surface; (ii) an inverse ratio II where the area loss decreases. The reduction in the degree of damage is associated with the reduction in the proportion of craters since the incremental rate of area loss caused by indentations and scratches is bigger than that caused by the craters; and (iii) a direct ratio III where the number of craters decrease and particle abrasion increases.

Comparing curves a and b, when the $0.5 \mu \mathrm{m}$ to $6.5 \mu \mathrm{m}$ microparticles were added to the wet steam the area loss of curve $b$ was higher than that of curve $a$. That is because the area of a crater is usually larger than that of an indentation and the area loss is mainly caused by the generation of craters, as shown in Figure 3(a) and (b). For microparticles larger than $6.5 \mu \mathrm{m}$ the area loss of both curves is nearly equal and increases rapidly. In this part, few craters appear on the surface and abrasion is the main type of damage with a further increase in microparticle size, as shown in Figure $3(c)$ and (d). Therefore, microparticle size has a significant effect on crater generation and the degree of erosion damage. 


\subsection{Effect of microparticle shape}

In this section, microparticles of similar size $(4 \mu \mathrm{m})$ and different shape (irregular or spherical) were added to wet steam to determine the effect of microparticle shape. Figure 5 shows the damaged surface after these experiments.

From Figure 5(a), the type of damage is identical to that from the experiment described in Section 3.1. As spherical microparticles were also added to the wet steam the eroded surface also shows indentations and craters but few scratches are present on the surface, as shown in Figure 5(b). The craters that come from the addition of irregular or spherical microparticles to the wet steam have the same characteristics such as circular and smooth brims. The brims are smoother and smaller for indentations than that caused by the irregular microparticles.

The scanning electron micrographs were also processed by pit identification and Figure 6 shows the relationship between area loss and microparticle shape.

Figure 6 shows that when the material surface is eroded by the addition of irregular microparticles or spherical microparticles to wet steam the area loss caused by craters is nearly equal, which indicates that the microparticle's shape hardly affects the generation of craters. These results seem to conflict with Harvey's theory [15] to some extent because the crevices and caves on the surface are usually thought to promote the growth of cavities. However, in the work of Arora et al. [16] large vapor cavities were observed to grow directly from small regions on the spherical particle surface using high speed photography. Therefore, cavities can grow from the microparticle's surface and is almost independent of the microparticle's shape. The shape is, therefore, not as important as the other microparticle properties such as its size as discussed in section 2.1.

The area loss caused by the irregular microparticles is larger than that caused by spherical microparticles. Because the two kinds of microparticle have the same hardness and size, the shape of the microparticle is the main factor that causes this difference. Because $\mathrm{SiC}$ microparticles are harder than AISI 1045 steel (the hardness of $\mathrm{SiC}$ is HV1800-2000 and AISI 1045 steel is HV650) the microparticles are like indenters when they impact the steel's surface. Because an indentation is more easily formed by a sharp triangular indenter than by a spherical indenter, irregular
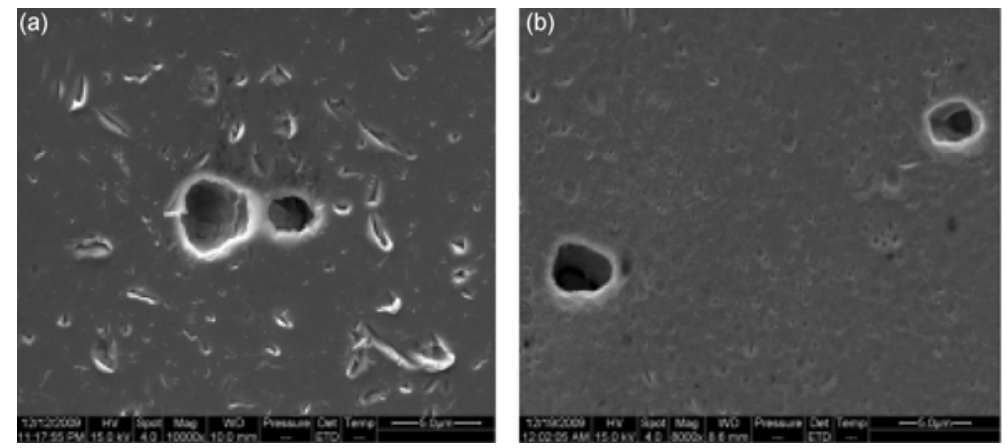

Figure 5 Scanning electron micrographs of the surfaces eroded by the addition of microparticles of different shape to the wet steam. (a) For irregular microparticles and (b) for spherical microparticles.

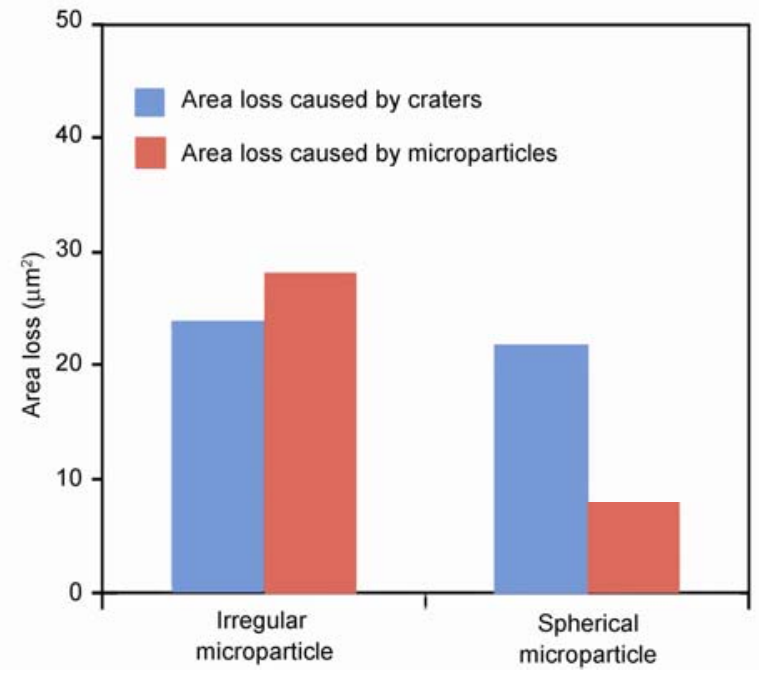

Figure 6 Relationship between area loss and microparticle shape. microparticles cause more severe damage than spherical microparticles.

\section{Conclusions}

(1) Microparticle size significantly affects crater generation and the degree of erosion damage. Because the main type of damage is different for crater generation compared with particle abrasion, the relationship between area loss and size can be understood as three ratios including a direct ratio I, an inverse ratio II and a direct ratio III with an increase in size.

(2) Microparticle shape hardly affects crater generation and the degree of damage induced by the microparticles differs. Compared with spherical microparticles, irregular microparticles cause more severe abrasion on material surfaces. 
1 Pollard D, Lord M J, Stockton E C. An evaluation of low pressure steam turbine blade erosion. GECJ Sci Technol, 1983, 1: 29-34

2 Dehouve J, Nardin P, Zeghmati M. Erosion study of final stage blading of low pressure steam turbines. Appl Surf Sci, 1999, 144-145: 238-243

3 Min K L, Whung W K, Chang K R, et al. Investigation of liquid impact erosion for 12Cr steel and Stellite 6B. J Nucl Mater, 1998, 257: 134-144

4 Mann B S, Arya V. An experimental study to correlate water jet impingement erosion resistance and properties of metallic materials and coatings. Wear, 2002, 253: 650-661

5 Rao J S. Application of fracture mechanics in the failure analysis of a last stage steam turbine blade. Mech Mach Theory, 1998, 33: 599-602

6 Stanisa B, Ivusic V. Erosion behaviour and mechanisms for steam turbine rotor blades. Wear, 1995, 186-187: 395-400

7 Mann B S. Solid-particle erosion and protective layers for steam turbine blading. Wear, 1999, 224: 8-12

8 Wang J D, Chen H S, Qin L, et al. The crucial effect of micro-particles in hydraulic machinery cavitation corrosion. Chinese Sci Bull, 2007, 52: 2683-2687
9 Xu W L, Qin L, Chen H S, et al. Erosion and abrasion on mild carbon steel surface by steam containing SiC microparticles. Wear, 2010, 268: 1547-1550

10 Li Y J. Study on mechanism of surface topograhpy effects on generation of cavitation erosion. Dissertation for the Doctoral Degree. Beijing: Tsinghua University, 2008

$11 \mathrm{Xu} \mathrm{W} \mathrm{L.} \mathrm{Research} \mathrm{on} \mathrm{mechanism} \mathrm{of} \mathrm{erosion} \mathrm{induced} \mathrm{by} \mathrm{wet} \mathrm{steam.} \mathrm{Dis-}$ sertation for the Doctoral Degree. Beijing: Tsinghua University, 2010

12 Kennedy C F, Field J E. Damage threshold velocities for liquid impact. J Mater Sci, 2000, 35: 5331-5339

13 Huang S, Ihara A, Watanabe H, et al. Effects of solid particle properties on cavitation erosion in solid-water mixtures. J Fluids Eng, 1996, 118: 749-755

14 Rickard G, Staffan J. The particle size effect in abrasion studied by controlled abrasive surfaces. Wear, 1999, 224: 118-225

15 Harvey, Newton E, Mceiroy W D, et al. On cavity formation in water. J Appl Phys, 1947, 18: 162-172

16 Arora M, Ohl C D, Morch K A. Cavitation inception on microparticles: A self-propelled particle accelerator. Phys Rev Lett, 2004, 92 : 174501-1-174501-4

Open Access This article is distributed under the terms of the Creative Commons Attribution License which permits any use, distribution, and reproduction in any medium, provided the original author(s) and source are credited. 